PROCEEDINGS OF THE

AMERICAN MATHEMATICAL SOCIETY

Volume 138, Number 9, September 2010, Pages 3219-3228

S 0002-9939(10)10440-7

Article electronically published on April 30, 2010

\title{
UNIFORM GLOBAL ATTRACTORS FOR FIRST ORDER NON-AUTONOMOUS LATTICE DYNAMICAL SYSTEMS
}

\author{
AHMED Y. ABDALLAH
}

(Communicated by Yingfei Yi)

\begin{abstract}
Recently, many authors investigated the existence of global attractors for different types of autonomous lattice dynamical systems. Within this work, we carefully study the existence of a uniform global attractor for a new class of first order non-autonomous lattice dynamical system in the Hilbert space $l^{2}$.
\end{abstract}

\section{INTRODUCTION}

Lattice dynamical systems (LDSs) occur in a wide variety of applications in science and engineering, for instance, in propagation of nerve pulses in myelinated axons [5, 6, 20, 21, electrical engineering [9, pattern recognition [11, 13, 24, image processing [14, 15, 16, chemical reaction theory [17, 19], etc. In each case, they have their own form, but in some cases, they appear as spatial discretizations of corresponding continuous partial differential equations (PDSs) on unbounded domains. The traveling solutions for LDSs have been examined by [4, 7, 12, 17, 29] and the chaotic properties or pattern formation properties of solutions for LDSs have been investigated by $11,13,24$.

The importance of studying the existence of the global attractor for a dissipative dynamical system came from the fact that the global attractor is the smallest compact set, with respect to inclusion, that is invariant, attracts all the trajectories originating from the whole phase space and sometimes it has finite dimension. However, the long-time behavior of solutions for continuous partial differential equations on unbounded domains raises some difficulty, such as well-posedness and lack of compactness of Sobolev embeddings for obtaining the existence of global attractors. Therefore it is significant to study the existence of global attractors for LDSs because of the importance of such systems, and they can be considered as an approximation to the corresponding continuous PDSs if they appear as spatial discretizations of such PDSs.

Many researchers studied the existence and upper semicontinuity of global attractors for various types of autonomous LDSs $1,2,2,3,8,26,27,28$. In 25, the author investigated the existence and upper semicontinuity of a uniform global attractor

Received by the editors December 15, 2009.

2010 Mathematics Subject Classification. Primary 37L30, 37L60.

Key words and phrases. Non-autonomous lattice dynamical system, uniform absorbing set, uniform global attractor. 
for a first order non-autonomous LDS. Within this work we carefully study the existence of a uniform global attractor for a new class of first order non-autonomous LDSs in the Hilbert space $l^{2}$.

\section{Preliminaries}

For any positive integer $k$, consider the Hilbert space

$$
l^{2}=\left\{u=\left(u_{i}\right)_{i \in \mathbb{Z}^{k}}: i=\left(i_{1}, i_{2}, \ldots, i_{k}\right) \in \mathbb{Z}^{k}, u_{i} \in \mathbb{R}, \sum_{i \in \mathbb{Z}^{k}} u_{i}^{2}<\infty\right\},
$$

whose inner product and norm are given by

$$
\langle u, v\rangle=\sum_{i \in \mathbb{Z}^{k}} u_{i} v_{i},\|u\|=\langle u, u\rangle^{1 / 2}, \forall u=\left(u_{i}\right)_{i \in \mathbb{Z}^{k}}, v=\left(v_{i}\right)_{i \in \mathbb{Z}^{k}} \in l^{2} .
$$

Let

$$
I_{i q}=\left\{j=\left(j_{1}, j_{2}, \ldots, j_{k}\right) \in \mathbb{Z}^{k}: \max _{1 \leq l \leq k}\left|i_{l}-j_{l}\right| \leq q\right\}, \forall i=\left(i_{1}, i_{2}, \ldots, i_{k}\right) \in \mathbb{Z}^{k} .
$$

Note that the definitions and the basic theory related to the existence of uniform global attractors are given within section 4 .

For $\tau \in \mathbb{R}$, we will investigate the existence of uniform global attractors for 1st order non-autonomous LDSs of the following form:

$$
\dot{u}_{i}(t)+(A u(t))_{i}+f_{i}\left(u_{j}(t): j \in I_{i q}\right)=g_{i}(t), \forall i \in \mathbb{Z}^{k}, t>\tau,
$$

with initial data

$$
u_{i}(\tau)=u_{i, \tau}, \forall i \in \mathbb{Z}^{k}
$$

which can be written in the abstract form

$$
\begin{gathered}
\dot{u}(t)+A u(t)+f(u(t))=g(t), t>\tau, \\
u(\tau)=\left(u_{i, \tau}\right)_{i \in \mathbb{Z}^{k}}=u_{\tau},
\end{gathered}
$$

where $u(t)=\left(u_{i}(t)\right)_{i \in \mathbb{Z}^{k}}, A u(t)=\left((A u(t))_{i}\right)_{i \in \mathbb{Z}^{k}}, f(u(t))=\left(f_{i}\left(u_{j}(t)\right.\right.$ : $\left.\left.j \in I_{i q}\right)\right)_{i \in \mathbb{Z}^{k}}, g(t)=\left(g_{i}(t)\right)_{i \in \mathbb{Z}^{k}}$.

Letting $g_{0}: \mathbb{R} \rightarrow l^{2}$ be an almost periodic function, from 22] we know that such a function is bounded and uniformly continuous on $\mathbb{R}$. Therefore $g_{0} \in C_{b}\left(\mathbb{R}, l^{2}\right)$, where $C_{b}\left(\mathbb{R}, l^{2}\right)$ is the space of bounded continuous functions on $\mathbb{R}$ with the norm

$$
\|g\|_{C_{b}}=\sup _{t \in \mathbb{R}}\|g(t)\|_{l^{2}}, \forall g \in C_{b}\left(\mathbb{R}, l^{2}\right) .
$$

Moreover, since $g_{0}: \mathbb{R} \rightarrow l^{2}$ is an almost periodic function, by Bochner's criterion 22], the set of translations $\left\{g_{0}(\cdot+h): h \in \mathbb{R}\right\}$ is precompact in $C_{b}\left(\mathbb{R}, l^{2}\right)$. Let $\mathcal{H}\left(g_{0}\right)=\overline{\left\{g_{0}(\cdot+h): h \in \mathbb{R}\right\}}$ in $C_{b}\left(\mathbb{R}, l^{2}\right)$. Then for any $g \in \mathcal{H}\left(g_{0}\right), g$ is almost periodic and $\mathcal{H}(g)=\mathcal{H}\left(g_{0}\right)$.

Recalling the LDS (2.3) - (2.4), within this work we assume that:

$(A 1) g \in \mathcal{H}\left(g_{0}\right)$, where $g_{0}: \mathbb{R} \rightarrow l^{2}$ is an almost periodic function.

(A2) $A: l^{2} \rightarrow l^{2}$ is a bounded linear operator of the following form:

$$
A=A_{1}+A_{2}+\ldots+A_{k}
$$


and there exist bounded linear operators $D_{j}: l^{2} \rightarrow l^{2}$ given by

$$
\left(D_{j} u\right)_{i}=\sum_{l=-m_{0}}^{m_{0}} d_{j, l} u_{i_{j, l}}, \forall u=\left(u_{i}\right)_{i \in \mathbb{Z}^{k}} \in l^{2}, j=1,2, \ldots, k,
$$

where $d_{j, l} \in \mathbb{R}$ such that not all of them are zeros, $m_{0}$ is a positive integer, $i_{j, l}=$ $\left(i_{1}, \ldots, i_{j-1}, i_{j}+l, i_{j+1}, \ldots, i_{k}\right) \in \mathbb{Z}^{k}$, such that

$$
A_{j}=D_{j}^{*} D_{j}=D_{j} D_{j}^{*},\left\|D_{j}\right\|_{O} \leq c_{0}, \forall j=1,2, \ldots, k,
$$

where $\|\cdot\|_{O}$ denotes the norm of an operator in the set of linear operators from $l^{2}$ into itself, $c_{0} \geq 0$ and $D_{j}^{*}$ is the adjoint operator of $D_{j}$, that is,

$$
\left(D_{j}^{*} u\right)_{i}=\sum_{l=-m_{0}}^{m_{0}} d_{j,-l} u_{i_{j, l}},\left\langle D_{j} u, v\right\rangle=\left\langle u, D_{j}^{*} v\right\rangle, \forall u=\left(u_{i}\right)_{i \in \mathbb{Z}^{k}}, v \in l^{2}, j=1,2, \ldots, k .
$$

(A3) $f$ is locally Lipschitz continuous from $l^{2}$ into $l^{2}$ and there exist positive constants $\xi, I_{0}$ and $c_{1}$ such that for $i \in \mathbb{Z}^{k}$,

$$
\begin{gathered}
f_{i}\left(u_{j}: j \in I_{i q}\right) u_{i} \geq 0, \forall \sum_{j \in I_{i q}}\left|u_{j}\right|>\xi,\|i\|_{m} \leq I_{0}, \\
f_{i}\left(u_{j}: j \in I_{i q}\right) u_{i} \geq c_{1} u_{i}^{2}, \forall u_{j} \in \mathbb{R},\|i\|_{m}>I_{0},
\end{gathered}
$$

where

$$
\|i\|_{m}=\max _{1 \leq l \leq k}\left|i_{l}\right|, \forall i=\left(i_{1}, i_{2}, \ldots, i_{k}\right) \in \mathbb{Z}^{k}
$$

Remark 1. Here we connect the LDS (2.3) - 2.4), given above, with the LDSs presented in [25, 27. In fact, we introduced weaker conditions on the nonlinear part of the system as we will see within this remark. For each $i \in \mathbb{Z}^{k}$, let $f_{i}\left(u_{j}: j \in I_{i q}\right)=\lambda_{i} u_{i}+F_{i}\left(u_{j}: j \in I_{i q}\right)$, where $\lambda_{0} \leq \lambda_{i} \leq \lambda^{0}$ for some $\lambda_{0}, \lambda^{0}>$ 0 . Then the LDS (2.3) - (2.4) will be of the form

$$
\begin{gathered}
\dot{u}_{i}(t)+(A u(t))_{i}+\lambda_{i} u_{i}(t)+F_{i}\left(u_{j}: j \in I_{i q}\right)=g_{i}(t), \forall i \in \mathbb{Z}^{k}, t>\tau, \\
u(\tau)=\left(u_{i, \tau}\right)_{i \in \mathbb{Z}^{k}}=u_{\tau},
\end{gathered}
$$

with

$$
\begin{gathered}
F_{i}\left(u_{j}: j \in I_{i q}\right) u_{i} \geq-\lambda_{i} u_{i}^{2}, \forall \sum_{j \in I_{i q}}\left|u_{j}\right|>\xi,\|i\|_{m} \leq I_{0}, \\
F_{i}\left(u_{j}: j \in I_{i q}\right) u_{i} \geq\left(c_{1}-\lambda_{i}\right) u_{i}^{2}, \forall u_{j} \in \mathbb{R},\|i\|_{m}>I_{0},
\end{gathered}
$$

where we can choose $c_{1} \leq \lambda_{0}$. In such a case, if we consider $\tau=0$ and $\left(g_{i}(t)\right)_{i \in \mathbb{Z}^{k}}=$ $\left(g_{i}\right)_{i \in \mathbb{Z}^{k}} \in l^{2}, \forall t \geq 0$, then the LDS (2.11) - (2.12) is consistent with the autonomous LDS (14) of [27, where it has been assumed that

$$
F_{i}\left(u_{j}: j \in I_{i q}\right) u_{i} \geq 0, \forall u_{j} \in \mathbb{R}, i \in \mathbb{Z}^{k},
$$

and the existence and upper semicontinuity of global attractors for such autonomous LDSs have been studied. Moreover, with $q=0$ and $F_{i}=F, \forall i \in \mathbb{Z}^{k}$, the LDS (2.11) - (2.12) is consistent with the non-autonomous $\operatorname{LDS}(2.1)-(2.2)$ of [25], where it has been assumed that

$$
F(s) s \geq 0, \forall s \in \mathbb{R},
$$

and the existence and upper semicontinuity of uniform global attractors for such non-autonomous LDSs have been investigated. 


\section{Global solutions And ABSORBing SETS}

Following Lemma 2 of [2], here we prove the following lemma, which is the key idea for completing our work.

Lemma 1. There exist positive constants $c_{2}$ and $c_{3}$ such that

$$
\sum_{j=1}^{k}\left\|D_{j} u\right\|^{2}+\langle f(u), u\rangle \geq c_{3}\|u\|^{2}-c_{2}, \forall u=\left(u_{i}\right)_{i \in \mathbb{Z}^{k}} \in l^{2} .
$$

Proof. Using (2.9) - (2.10), there exists $c_{2}>0$ such that

$$
\langle f(u), u\rangle \geq c_{1} \sum_{\|i\|_{m}>I_{0}} u_{i}^{2}-c_{2}
$$

In such a case it is enough to show that there exists $c_{3}>0$ such that

$$
\sum_{j=1}^{k}\left\|D_{j} u\right\|^{2}+\left(c_{1}-c_{3}\right) \sum_{\|i\|_{m}>I_{0}} u_{i}^{2} \geq c_{3} \sum_{\|i\|_{m} \leq I_{0}} u_{i}^{2} .
$$

Recalling (2.6), we assumed that there exist $j_{0} \in\{1, \ldots, k\}$ and $l_{0} \in\left\{-m_{0}, \ldots, m_{0}\right\}$ such that $d_{j_{0}, l_{0}} \neq 0$. Without loss of generality we can assume that $l_{0}$ is the smallest integer between $-m_{0}$ and $m_{0}$ such that $d_{j_{0}, l_{0}} \neq 0$. For $i \in \mathbb{Z}^{k}$, with $\|i\|_{m} \leq I_{0}$, we have

$$
\begin{aligned}
& u_{i}^{2}=\frac{1}{d_{j_{0}, l_{0}}^{2}}\left(\sum_{l_{1}=l_{0}}^{m_{0}} d_{j_{0}, l_{1}} u_{i_{j_{0}, l_{1}-l_{0}}}-\sum_{l_{1}=l_{0}+1}^{m_{0}} d_{j_{0}, l_{1}} u_{i_{j_{0}, l_{1}-l_{0}}}\right)^{2} \\
\leq & \frac{2}{d_{j_{0}, l_{0}}^{2}}\left(\left(\sum_{l_{1}=l_{0}}^{m_{0}} d_{j_{0}, l_{1}} u_{i_{j_{0}, l_{1}-l_{0}}}\right)^{2}+\left(\sum_{l_{1}=1}^{m_{0}-l_{0}} d_{j_{0}, l_{1}+l_{0}} u_{i_{j_{0}, l_{1}}}\right)^{2}\right) \\
\leq & \frac{2}{d_{j_{0}, l_{0}}^{2}}\left(\left(D_{j_{0}} u\right)_{i_{j_{0},-l_{0}}}^{2}+\sum_{l_{1}=1}^{m_{0}-l_{0}}\left(2^{l_{1}}\left(d_{j_{0}, l_{1}+l_{0}} u_{i_{j_{0}, l_{1}}}\right)^{2}\right)\right) .
\end{aligned}
$$

Similarly, for each $l_{1}=1,2, \ldots, m_{0}-l_{0}$, we have

$$
u_{i_{j_{0}, l_{1}}}^{2} \leq \frac{2}{d_{j_{0}, l_{0}}^{2}}\left(\left(D_{j_{0}} u\right)_{i_{j_{0}, l_{1}-l_{0}}}^{2}+\sum_{l_{2}=1}^{m_{0}-l_{0}}\left(2^{l_{2}}\left(d_{j_{0}, l_{2}+l_{0}} u_{i_{j_{0}, l_{1}+l_{2}}}\right)^{2}\right)\right),
$$

and for each $l_{p}=1,2, \ldots, m_{0}-l_{0}, p=1,2$, we have

$$
u_{i_{j_{0}, l_{1}+l_{2}}}^{2} \leq \frac{2}{d_{j_{0}, l_{0}}^{2}}\left(\left(D_{j_{0}} u\right)_{i_{j_{0}, l_{1}+l_{2}-l_{0}}}^{2}+\sum_{l_{3}=1}^{m_{0}-l_{0}}\left(2^{l_{3}}\left(d_{j_{0}, l_{3}+l_{0}} u_{i_{j_{0}, l_{1}+l_{2}+l_{3}}}\right)^{2}\right)\right) .
$$

Following the same procedure which is used in 3.3 - 3.5 , it follows that for each positive integer $q$ and $l_{p}=1,2, \ldots, m_{0}-l_{0}, p=1,2, \ldots, q$,

$$
u_{i_{j_{0}, l_{1}+\ldots+l_{q}}}^{2} \leq \frac{2}{d_{j_{0}, l_{0}}^{2}}\left(+\sum_{l_{q+1}=1}^{m_{0}-l_{0}}\left(2^{l_{q+1}}\left(d_{j_{0}, l_{q+1}+l_{0}} u u_{i_{j_{j_{0}, l_{1}, \ldots+l_{1}+\ldots+1}}^{2}}^{2}\right)^{2}\right)\right) .
$$

In fact we need to apply inequality (3.6) only for $\left\|i_{j_{0}, l_{1}+\ldots+l_{q}}\right\|_{m} \leq I_{0}$. Hence from (3.3) - (3.6), it is clear that for sufficiently small $c_{3}$, inequality (3.2) is correct. 
From assumptions (A2) and (A3), we know that $f: l^{2} \rightarrow l^{2}$ is locally Lipschitz continuous and $A: l^{2} \rightarrow l^{2}$ is a bounded linear operator. In such a case, following the standard theory of ordinary differential equations, for $g \in \mathcal{H}\left(g_{0}\right), \tau \in \mathbb{R}$, and $u_{\tau} \in l^{2}$, there exists a unique local maximal solution $u$ of (2.3) - (2.4) in $l^{2}$ such that $u \in C\left([\tau, T), l^{2}\right) \cap C^{1}\left((\tau, T), l^{2}\right)$, for some $T>0$. Moreover, if $T<\infty$, then $\lim _{t \rightarrow T^{-}}\|u(t)\|_{E}^{2}=\infty$.

Lemma 2. Given $g \in \mathcal{H}\left(g_{0}\right), \tau \in \mathbb{R}$, and $u_{\tau} \in l^{2}$, the solution $u$ of (2.3) - (2.4) in $l^{2}$ satisfies

$$
\|u(t)\|^{2} \leq e^{c_{3}(\tau-t)}\left\|u_{\tau}\right\|^{2}+\left(1-e^{c_{3}(\tau-t)}\right)\left(\frac{1}{c_{3}^{2}}\left\|g_{0}\right\|_{C_{b}}^{2}+\frac{2 c_{2}}{c_{3}}\right), \forall t \geq \tau .
$$

Proof. Considering the inner product of (2.3) with $u(t)$ in $l^{2}$, we obtain that

$$
\frac{1}{2} \frac{d}{d t}\|u(t)\|^{2}+\sum_{j=1}^{k}\left\|D_{j} u(t)\right\|^{2}+\langle f(u(t)), u(t)\rangle=\langle g(t), u(t)\rangle, \forall t>\tau .
$$

By (3.1),

$$
\frac{1}{2} \frac{d}{d t}\|u(t)\|^{2}+c_{3}\|u(t)\|^{2}-c_{2} \leq\langle g(t), u(t)\rangle \leq \frac{1}{2 c_{3}}\|g(t)\|^{2}+\frac{c_{3}}{2}\|u(t)\|^{2}, \forall t>\tau .
$$

Recalling assumption $(A 1)$, we get

$$
\begin{gathered}
\frac{d}{d t}\|u(t)\|^{2}+c_{3}\|u(t)\|^{2} \leq \frac{1}{c_{3}}\left\|g_{0}\right\|_{C_{b}}^{2}+2 c_{2}, \forall t>\tau, \\
\frac{d}{d t}\left(e^{c_{3} t}\|u(t)\|^{2}\right) \leq e^{c_{3} t}\left(\frac{1}{c_{3}}\left\|g_{0}\right\|_{C_{b}}^{2}+2 c_{2}\right), \forall t>\tau .
\end{gathered}
$$

Integrating the last inequality from $\tau$ into $t$, we get (3.7) .

Following (3.7), we find that the solution $u$ of (2.3) - (2.4) is defined globally for $t \geq \tau$. In such a case we can introduce a family of processes

$$
\left\{U^{g}(t, \tau): t \geq \tau, \tau \in \mathbb{R}\right\}_{g \in \mathcal{H}\left(g_{0}\right)}
$$

on $l^{2}$ such that for $g \in \mathcal{H}\left(g_{0}\right), \tau \in \mathbb{R}, t \geq \tau$, and $u_{\tau} \in l^{2}$, we have $U^{g}(t, \tau) u_{\tau}=u(t)$, where $u$ is the solution of (2.3) - (2.4). By the unique solvability of (2.3) - (2.4), the family of processes $\left\{U^{g}(t, \tau)\right\}_{g \in \mathcal{H}\left(g_{0}\right)}$ satisfies the multiplicative properties

$$
\begin{gathered}
U^{g}(t, s) U^{g}(s, \tau)=U^{g}(t, \tau), \forall t \geq s \geq \tau, \tau \in \mathbb{R}, \\
U^{g}(\tau, \tau)=I, \forall \tau \in \mathbb{R},
\end{gathered}
$$

where $I$ is the identity operator. Moreover the following translation identity holds for the processes and the translation group $\{T(h)\}_{h \in \mathbb{R}}$ :

$$
U^{g}(t+h, \tau+h)=U^{T(h) g}(t, \tau), \forall h, \tau \in \mathbb{R}, t \geq \tau,
$$

where

$$
T(h) g=g(\cdot+h), \forall g \in \mathcal{H}\left(g_{0}\right) .
$$

Along the lines of (3.7), we get the following lemma. 
Lemma 3. In $l^{2}$, the closed bounded ball $\mathcal{B}=\mathcal{B}\left(0, R_{0}\right)$ with center 0 and radius $R_{0}>\sqrt{\frac{1}{c_{3}^{2}}\left\|g_{0}\right\|_{C_{b}}^{2}+\frac{2 c_{2}}{c_{3}}}$ is a uniform absorbing set for the family of processes $\left\{U^{g}(t, \tau)\right\}_{g \in \mathcal{H}\left(g_{0}\right)}$ corresponding to the LDS (2.3) - (2.4) with respect to $g \in \mathcal{H}\left(g_{0}\right)$. That is, for $\tau \in \mathbb{R}$ and any bounded set $G$, there exists a constant $T=T\left(G, R_{0}\right) \geq 0$ such that

$$
U^{g}(t, \tau) G \subseteq \mathcal{B}, \forall g \in \mathcal{H}\left(g_{0}\right), t-\tau \geq T
$$

Moreover,

$$
U^{g}(t, \tau) \mathcal{B} \subseteq \mathcal{B}, \forall g \in \mathcal{H}\left(g_{0}\right), t-\tau \geq 0 .
$$

\section{UNIFORM GLOBAL ATTRACTORS}

Lemma 4. Given $\eta>0$, there exist constants $I=I(\eta)$ and $T=T(\eta)$ such that for $g \in \mathcal{H}\left(g_{0}\right), \tau \in \mathbb{R}, t \geq \tau$, and $u_{\tau} \in \mathcal{B}$, the solution $u$ of (2.3) - (2.4) satisfies

$$
\sum_{\|i\|_{m} \geq I}\left(u_{i}(t)\right)^{2} \leq \eta, \forall t-\tau \geq T .
$$

Proof. Consider a smooth increasing function $\theta \in C^{1}\left(\mathbb{R}^{+}, \mathbb{R}\right)$ such that

$$
\left\{\begin{array}{cc}
\theta(s)=0, & 0 \leq s<1 \\
0 \leq \theta(s) \leq 1, & 1 \leq s<2, \\
\theta(s)=1, & 2 \leq s,
\end{array}\right.
$$

and there exists a constant $M_{0}$ such that $\left|\theta^{\prime}(s)\right| \leq M_{0}, \forall s \geq 0$. Since $g$ is almost periodic, the set $\left\{\left(g_{i}(t)\right)_{i \in \mathbb{Z}^{k}}: t \in \mathbb{R}\right\}$ is precompact in $l^{2}$. Moreover $g \in \mathcal{H}\left(g_{0}\right)$, and $\mathcal{H}\left(g_{0}\right)$ is compact in $C_{b}\left(\mathbb{R}, l^{2}\right)$. In such a case, given $\eta>0$, there exists a constant $M_{1}=M_{1}(\eta)$ (of course $M_{1}$ depends on $g_{0}$ but it is independent of $g$ ) such that

$$
\sum_{\|i\|_{m} \geq M_{1}} g_{i}^{2}(t) \leq \frac{\eta c_{1}^{2}}{4}, \forall t \in \mathbb{R},
$$

where $c_{1}$ is given by (2.10).

Let us choose $M_{2}=M_{2}(\eta)$ to be a positive integer such that

$$
M_{2}=\max \left\{I_{0}, M_{1}, \frac{32 k R_{0}^{2} M_{0} m_{0}^{2} c_{0}^{2}}{\eta c_{1}}\right\},
$$

and for each $i \in \mathbb{Z}^{k}$, let $z_{i}=\theta\left(\frac{\|i\|_{m}}{M_{2}}\right) u_{i}$ and $z=\left(z_{i}\right)_{i \in \mathbb{Z}^{k}}$. Taking the inner product of (2.3) with $z$ in $l^{2}$, we obtain that

$$
\begin{gathered}
\frac{1}{2} \sum_{i \in \mathbb{Z}^{k}} \theta\left(\frac{\|i\|_{m}}{M_{2}}\right) \frac{d}{d t} u_{i}^{2}(t)+\sum_{j=1}^{k}\left\langle D_{j} u(t), D_{j} z(t)\right\rangle \\
+\sum_{i \in \mathbb{Z}^{k}} \theta\left(\frac{\|i\|_{m}}{M_{2}}\right) f_{i}\left(u_{j}(t): j \in I_{i q}\right) u_{i}(t) \\
=\sum_{i \in \mathbb{Z}^{k}} \theta\left(\frac{\|i\|_{m}}{M_{2}}\right) g_{i}(t) u_{i}(t), \forall t-\tau \geq 0 .
\end{gathered}
$$

Using (2.10), (4.1) and (4.3), we get

$$
\sum_{i \in \mathbb{Z}^{k}} \theta\left(\frac{\|i\|_{m}}{M_{2}}\right) f_{i}\left(u_{j}(t): j \in I_{i q}\right) u_{i}(t) \geq c_{1} \sum_{i \in \mathbb{Z}^{k}} \theta\left(\frac{\|i\|_{m}}{M_{2}}\right) u_{i}^{2}(t), \forall t-\tau \geq 0,
$$


and by (4.1) - 4.3), we find that

$$
\begin{gathered}
\sum_{i \in \mathbb{Z}^{k}} \theta\left(\frac{\|i\|_{m}}{M_{2}}\right) g_{i}(t) u_{i}(t) \leq \frac{1}{2 c_{1}} \sum_{i \in \mathbb{Z}^{k}} \theta\left(\frac{\|i\|_{m}}{M_{2}}\right) g_{i}^{2}(t) \\
+\frac{c_{1}}{2} \sum_{i \in \mathbb{Z}^{k}} \theta\left(\frac{\|i\|_{m}}{M_{2}}\right) u_{i}^{2}(t) \leq \frac{\eta c_{1}}{8}+\frac{c_{1}}{2} \sum_{i \in \mathbb{Z}^{k}} \theta\left(\frac{\|i\|_{m}}{M_{2}}\right) u_{i}^{2}(t), \forall t-\tau \geq 0 .
\end{gathered}
$$

Following (31) of [27] and using (3.11), given above, we get

$$
\begin{gathered}
\left\langle D_{j} u(t), D_{j} z(t)\right\rangle=\sum_{i \in \mathbb{Z}^{k}} \theta\left(\frac{\|i\|_{m}}{M_{2}}\right)\left(D_{j} u(t)\right)_{i}^{2} \\
+\sum_{i \in \mathbb{Z}^{k}}\left(D_{j} u(t)\right)_{i}\left(\left(D_{j} z(t)\right)_{i}-\theta\left(\frac{\|i\|_{m}}{M_{2}}\right)\left(D_{j} u(t)\right)_{i}\right) \\
\geq \sum_{i \in \mathbb{Z}^{k}} \theta\left(\frac{\|i\|_{m}}{M_{2}}\right)\left(D_{j} u(t)\right)_{i}^{2}-\frac{4 R_{0}^{2} M_{0} m_{0}^{2} c_{0}^{2}}{M_{2}}, \forall j=1,2, \ldots, k, t-\tau \geq 0 .
\end{gathered}
$$

In such a case, by (4.3), we find that

$$
\left\langle D_{j} u(t), D_{j} z(t)\right\rangle \geq-\frac{\eta c_{1}}{8 k}, \forall j=1,2, \ldots, k, t-\tau \geq 0 .
$$

Recalling (4.4) - (4.7), it follows that

$$
\sum_{i \in \mathbb{Z}^{k}} \theta\left(\frac{\|i\|_{m}}{M_{2}}\right) \frac{d}{d t} u_{i}^{2}(t)+c_{1} \sum_{i \in \mathbb{Z}^{k}} \theta\left(\frac{\|i\|_{m}}{M_{2}}\right) u_{i}^{2}(t) \leq \frac{\eta c_{1}}{2}, \forall t-\tau \geq 0 .
$$

That is,

$$
\sum_{i \in \mathbb{Z}^{k}} \theta\left(\frac{\|i\|_{m}}{M_{2}}\right) \frac{d}{d t}\left(e^{c_{1} t} u_{i}^{2}(t)\right) \leq \frac{\eta c_{1}}{2} e^{c_{1} t}, \forall t-\tau \geq 0 .
$$

Integrating the last inequality from $\tau$ into $t$, we get

$$
\begin{gathered}
\sum_{i \in \mathbb{Z}^{k}} \theta\left(\frac{\|i\|_{m}}{M_{2}}\right) u_{i}^{2}(t) \leq e^{c_{1}(\tau-t)} \sum_{i \in \mathbb{Z}^{k}} \theta\left(\frac{\|i\|_{m}}{M_{2}}\right) u_{i}^{2}(\tau)+\frac{\eta}{2} \\
\leq e^{c_{1}(\tau-t)} R_{0}^{2}+\frac{\eta}{2}, \forall t-\tau \geq 0 .
\end{gathered}
$$

Thus for $I=I(\eta)=2 M_{2}(\eta)$ and $T=T(\eta)=\frac{1}{c_{1}} \ln \frac{2 R_{0}^{2}}{\eta}$, we have

$$
\sum_{\|i\|_{m} \geq I}\left(u_{i}(t)\right)^{2} \leq \sum_{i \in \mathbb{Z}^{k}} \theta\left(\frac{\|i\|_{m}}{M_{2}}\right) u_{i}^{2}(t) \leq \eta, \forall t-\tau \geq T .
$$

In $l^{2}$, let us connect the family of processes $\left\{U^{g}(t, \tau): t \geq \tau, \tau \in \mathbb{R}\right\}_{g \in \mathcal{H}\left(g_{0}\right)}$ corresponding to the LDS (2.3) - (2.4) with an associated semigroup of nonlinear operators, where we use the semigroup theory to investigate the existence of the uniform global attractor of the processes. Along the lines of [10, we define the nonlinear semigroup $\{\mathcal{S}(t)\}_{t>0}$ associated with the LDS (2.3) - (2.4) acting on the extended phase space $l^{2} \times \mathcal{H}\left(g_{0}\right)$ by

$$
\mathcal{S}(t)(u, g)=\left(U^{g}(t, 0) u, T(t) g\right), \forall t \geq 0, u \in l^{2}, g \in \mathcal{H}\left(g_{0}\right) .
$$

In such a case, we find that $\{\mathcal{S}(t)\}_{t \geq 0}$ satisfies the semigroup identities

$$
\mathcal{S}(t) \mathcal{S}(s)=\mathcal{S}(t+s), \mathcal{S}(0)=I, \forall t \geq s \geq 0 .
$$

Lemma 5. The solution semigroup $\{\mathcal{S}(t)\}_{t \geq 0}$ associated with the $L D S(2.3)-(2.4)$ is asymptotically compact; that is, if $\left\{\left(u_{n}, g_{n}\right)\right\}_{n=1}^{\infty}$ is bounded in $l^{2} \times \mathcal{H}\left(g_{0}\right)$, and $t_{n} \rightarrow \infty$, then $\left\{\mathcal{S}\left(t_{n}\right)\left(u_{n}, g_{n}\right)\right\}_{n=1}^{\infty}$ is precompact in $l^{2} \times \mathcal{H}\left(g_{0}\right)$.

Proof. Using Lemmas 3 and 4 above, the proof is similar to that of Lemma 5.4 25. 
Definition 1. In $l^{2}$, a closed set $\mathcal{A}$ is called the uniform attractor for the family of processes $\left\{U^{g}(t, \tau)\right\}_{g \in \mathcal{H}\left(g_{0}\right)}$ with respect to $g \in \mathcal{H}\left(g_{0}\right)$ if:

(a) For any bounded set $G \subset l^{2}$,

$$
\lim _{t \rightarrow \infty} \sup _{g \in \mathcal{H}\left(g_{0}\right)} \operatorname{dist}\left(U^{g}(t, \tau) G, \mathcal{A}\right)=0, \forall \tau \in \mathbb{R} .
$$

(b) (Minimal property) If $\overline{\mathcal{A}}$ is any closed subset of $l^{2}$ satisfying property (a), then $\mathcal{A} \subseteq \overline{\mathcal{A}}$.

Definition 2. Given $g \in \mathcal{H}\left(g_{0}\right)$, a curve $t \rightarrow u(t) \in l^{2}$ is said to be a complete solution for the process $U^{g}(t, \tau)$ if it satisfies

$$
U^{g}(t, \tau) u(\tau)=u(t), \forall \tau \in \mathbb{R}, t \geq \tau .
$$

The kernel of the process $U^{g}(t, \tau)$ is the collection $\mathcal{K}_{g}$ of all its bounded complete solutions, that is,

$$
\mathcal{K}_{g}=\left\{u(\cdot) \in C_{b}\left(\mathbb{R}, l^{2}\right): u(\cdot) \text { satisfies (4.9) }\right\} .
$$

The kernel section of the process $U^{g}(t, \tau)$ at time $s \in \mathbb{R}$ is the set

$$
\mathcal{K}_{g}(s)=\left\{u(s): u(\cdot) \in \mathcal{K}_{g}\right\} .
$$

Let $\mathcal{F}_{1}$ and $\mathcal{F}_{2}$ be the projectors of $l^{2} \times \mathcal{H}\left(g_{0}\right)$ onto $l^{2}$ and $\mathcal{H}\left(g_{0}\right)$, respectively. Following the uniform attractor theory [10, we have the following proposition.

Proposition 1. In $l^{2} \times \mathcal{H}\left(g_{0}\right)$, if the semigroup $\{\mathcal{S}(t)\}_{t \geq 0}$ is continuous, point dissipative, and asymptotically compact, then it has a compact global attractor $\mathcal{A}_{\mathcal{S}}$. Furthermore, in $l^{2}, \mathcal{A}=\mathcal{F}_{1} \mathcal{A}_{\mathcal{S}}$ is the compact uniform attractor for the family of processes $\left\{U^{g}(t, \tau)\right\}_{g \in \mathcal{H}\left(g_{0}\right)}$. In addition,

(a) $\mathcal{A}_{\mathcal{S}}=\bigcup_{g \in \mathcal{H}\left(g_{0}\right)} \mathcal{K}_{g}(0) \times\{g\}$,

(b) $\mathcal{A}=\bigcup_{g \in \mathcal{H}\left(g_{0}\right)} \mathcal{K}_{g}(0)$,

(c) $\mathcal{F}_{2} \mathcal{A}_{\mathcal{S}}=\mathcal{H}\left(g_{0}\right)$.

Theorem 1. In $l^{2}$, the family of processes $\left\{U^{g}(t, \tau)\right\}_{g \in \mathcal{H}\left(g_{0}\right)}$ associated with the $L D S$ (2.3) - (2.4) has a compact uniform attractor $\mathcal{A}$ with respect to $g \in \mathcal{H}\left(g_{0}\right)$.

Proof. Following Lemma 3.2 in [25], we find that the family of processes consists of processes continuous from $l^{2} \times \mathcal{H}\left(g_{0}\right)$ into $l^{2}$. In such a case, using the continuity of the translation group $\{T(t)\}_{t \in \mathbb{R}}$, it follows that the solution semigroup $\{\mathcal{S}(t)\}_{t \geq 0}$ associated with the LDS (2.3) - (2.4) is continuous in $l^{2} \times \mathcal{H}\left(g_{0}\right)$. Let $\mathcal{B}_{\mathcal{S}}=$ $\mathcal{B} \times \mathcal{H}\left(g_{0}\right)$, where $\mathcal{B}$ is the uniform absorbing set of the processes $\left\{U^{g}(t, \tau)\right\}_{g \in \mathcal{H}\left(g_{0}\right)}$ given by Lemma 3 . Then $\mathcal{B}_{\mathcal{S}}$ is a bounded absorbing set for the solution semigroup $\{\mathcal{S}(t)\}_{t \geq 0}$ in $l^{2} \times \mathcal{H}\left(g_{0}\right)$. From Proposition 1, taking into account Lemma 5, there exists a compact global attractor $\mathcal{A}_{\mathcal{S}}$ for the solution semigroup $\{\mathcal{S}(t)\}_{t \geq 0}$ in $l^{2} \times$ $\mathcal{H}\left(g_{0}\right)$ and $\mathcal{A}=\mathcal{F}_{1} \mathcal{A}_{\mathcal{S}}$ is the compact uniform global attractor for the family of processes $\left\{U^{g}(t, \tau)\right\}_{g \in \mathcal{H}\left(g_{0}\right)}$ in $l^{2}$ with respect to $g \in \mathcal{H}\left(g_{0}\right)$.

Remark 2. The technique of studying the upper semicontinuity of global attractors for infinite-dimensional autonomous LDSs [1, 2, 3, 8, 26, 27, 28, and for infinitedimensional non-autonomous LDSs 25$]$ is the same. In such a case, one can easily prove the upper semicontinuity of the uniform global attractor $\mathcal{A}$ associated with the family of processes $\left\{U^{g}(t, \tau)\right\}_{g \in \mathcal{H}\left(g_{0}\right)}$ of (2.3) - (2.4). That is, the uniform global attractor $\mathcal{A}$ generated by the infinite-dimensional non-autonomous LDS (2.3) - (2.4) 
can be approached by the uniform global attractors of finite-dimensional truncated ordinary differential systems.

\section{REFERENCES}

[1] A.Y. Abdallah, Asymptotic behavior of the Klein-Gordon-Schrödinger lattice dynamical systems, Comm. Pure. Appl. Anal. 5 (2006), 55-69. MR2190776 (2006i:37168)

[2] A.Y. Abdallah, Upper semicontinuity of the attractor for a second order lattice dynamical system, Disc. Cont. Dyn. Sys-B 5 (2005), 899-916. MR2170215 (2006j:37093)

[3] A.Y. Abdallah, Upper semicontinuity of the attractor for lattice dynamical systems of partly dissipative reaction-diffusion systems, J. Appl. Math. 3 (2005), 273-288. MR2201975 (2007a:37097)

[4] V.S. Afraimovich, V.I. Nekorkin, Chaos of traveling waves in a discrete chain of diffusively coupled maps, Int. J. Bifurc. Chaos 4 (1994), 631-637. MR.1298079 (95h:92007)

[5] J. Bell, Some threshold results for models of myelinated nerves, Math. Biosci. 54 (1981), 181-190. MR630848 (83e:92015)

[6] J. Bell, C. Cosner, Threshold behavior and propagation for nonlinear differential-difference systems motivated by modeling myelinated axons, Quart. Appl. Math. 42 (1984), 1-14. MR736501 (85h:92018)

[7] P.W. Bates, X. Chen, A. Chmaj, Traveling waves of bistable dynamics of a lattice, SIAM J. Math. Anal. 35 (2003), 520-546. MR2001111 (2004k:37178)

[8] P.W. Bates, K. Lu, B. Wang, Attractors for lattice dynamical systems, Int. J. Bifurc. Chaos 11 (2001), 143-153. MR1815532 (2001m:37172)

[9] T.L. Carrol, L.M. Pecora, Synchronization in chaotic systems, Phys. Rev. Lett. 64 (1990), 821-824. MR.1038263 (92c:58082)

[10] V.V. Chepyzhov, M.I. Vishik, Attractors of nonautonomous dynamical systems and their dimension, J. Math. Pures Appl. 73 (1994), 279-333. MR.1273705 (95c:34106)

[11] S.N. Chow, J. Mallet-Paret, Pattern formation and spatial chaos in lattice dynamical systems: I, IEEE Trans. Circuits and Systems 42 (1995), 746-751. MR1363311 (97b:58074)

[12] S.N. Chow, J. Mallet-Paret, W. Shen, Traveling waves in lattice dynamical systems, J. Diff. Eqs. 149 (1998), 248-291. MR1646240(2000b:37092)

[13] S.N. Chow, J. Mallet-Paret, E.S. Van Vleck, Pattern formation and spatial chaos in spatially discrete evolution equations, Rand. Comput. Dyn. 4 (1996), 109-178. MR1402415 (97d:34049)

[14] L.O. Chua, T. Roska, The CNN paradigm, IEEE Trans. Circuits and Systems 40 (1993), $147-156$.

[15] L.O. Chua, L. Yang, Cellular neural networks: theory, IEEE Trans. Circuits and Systems 35 (1988), 1257-1272. MR960777 (91d:92005)

[16] L.O. Chua, L. Yang, Cellular neural networks: applications, IEEE Trans. Circuits and Systems 35 (1988), 1273-1290. MR960778 (91d:92006)

[17] T. Erneux, G. Nicolis, Propagating waves in discrete bistable reaction diffusion systems, Physica D 67 (1993), 237-244. MR 1234443 (94e:92009)

[18] J. Hale, "Asymptotic Behavior of Dissipative Systems," Mathematical Surveys and Monographs 25, Amer. Math. Soc., Providence, Rhode Island, 1988. MR941371 (89g:58059)

[19] R. Kapral, Discrete models for chemically reacting systems, J. Math. Chem. 6 (1991), 113163. MR 1101758

[20] J.P. Keener, Propagation and its failure in coupled systems of discrete excitable cells, SIAM J. Appl. Math. 47 (1987), 556-572. MR889639 (88k:92028)

[21] J.P. Keener, The effects of discrete gap junction coupling on propagation in myocardium, J. Theor. Biol. 148 (1991), 49-82.

[22] B.M. Levitan, V.V. Zhikov, "Almost Periodic Functions and Differential Equations," Cambridge Univ. Press, Cambridge, 1982. MR690064 (84g:34004)

[23] Q.F. Ma, S.H. Wang, C.K. Zhong, Necessary and sufficient conditions for the existence of global attractor for semigroup and application, Indiana Univ. Math. J. 51 (2002), 1541-1559. MR 1948459 (2003j:37137)

[24] J. Mallet-Paret, S.N. Chow, Pattern formation and spatial chaos in lattice dynamical systems: II, IEEE Trans. Circuits and Systems 42 (1995), 752-756. MR1363311(97b:58074) 
[25] B. Wang, Asymptotic behavior of non-autonomous lattice systems, J. Math. Anal. Appl. 331 (2007), 121-136. MR2305992 (2008d:37147)

[26] S. Zhou, Attractors for second order lattice dynamical systems, J. Diff. Eqs. 179 (2002), 605-624. MR:1885681 (2002k:37165)

[27] S. Zhou, Attractors for first order dissipative lattice dynamical systems, Physica D 178 (2003), 51-61. MR 1983188 (2004b:37178)

[28] S. Zhou, Attractors and approximations for lattice dynamical systems, J. Diff. Eqs. 200 (2004), 342-368. MR2052618 (2005c:37159)

[29] B. Zinner, Existence of traveling wavefront solutions for the discrete Nagumo equation, J. Diff. Eqs. 96 (1992), 1-27. MR1153307 (93b:34095)

Department of Mathematics, University of Jordan, Amman 11942, Jordan

E-mail address: farah@ju.edu.jo 